\title{
Switching the Chirality of the Metal Environment Alters the Coordination Mode in Designed Peptides**
}

\author{
Anna F. A. Peacock, Jeanne A. Stuckey, and Vincent L. Pecoraro*
}

De novo metallopeptide design contributes significantly to the understanding of protein folding, protein-protein interactions, and metal-ion sites in biology. ${ }^{[1,2]}$ Within this rubric, studies in our research group have been focused on the biochemistry of $\mathrm{Cd}^{\mathrm{II}}$ thiolate sites. A class of peptides based on the parent peptide TRI, Ac-G(LKALEEK) ${ }_{4} \mathrm{G}-\mathrm{NH}_{2}$, was designed to assemble in aqueous solution into amphiphilic $\alpha$-helices. These peptides aggregate to form three-stranded coiled coils above $\mathrm{pH}$ 5.5. ${ }^{[3]}$ Substitutions can be made to the interior of these coiled coils to generate metal-binding sites, potentially with adjacent cavities. ${ }^{[4]}$ One benefit of de novo design is that we are no longer limited to the 20 naturally coded amino acids. We demonstrated by two different strategies that it is possible to create a coordinatively unsaturated trigonal-planar $\mathrm{CdS}_{3}$ site by incorporating nonprotein amino acids into the peptide sequence. The first approach was to replace the coordinating L-cysteine (L-Cys) residue with the bulkier nonprotein analogue $\mathrm{L}$-penicillamine (L-Pen), in which the $\beta$-methylene hydrogen atoms have been replaced with larger methyl groups. ${ }^{[8]}$ Our second approach was to modify the chirality of the second-coordination-sphere ligands directly above the metal-binding plane (L-Leu $\rightarrow$ D-Leu). In this way, the Leu side chain was reoriented towards the $\mathrm{C}$ terminus and the metal-binding site. Presumably, this modification sterically inhibits the binding of a fourth ligand to the $\mathrm{Cd}^{\mathrm{II}}$ center. ${ }^{[9,10]}$

Although these accomplishments were significant, we soon realized that we neither understood how L-Pen was capable of enforcing a $\mathrm{CdS}_{3}$ coordination sphere, nor how the introduction of a single layer of D-amino acids in the interior of a coiled coil composed of L-amino acids would affect the structure. We also realized that we could now assess how metal binding would be perturbed by combining increased steric bulk and alternate chirality within the same amino acid. One might expect that the simple replacement of an $\mathrm{L}$ with a D ligand would lead to the same coordination geometry with an inverted stereochemical configuration; however, since

[*] Dr. A. F. A. Peacock, Prof. V. L. Pecoraro

Department of Chemistry, University of Michigan

Ann Arbor, MI 48109 (USA)

Fax: $(+1)$ 734-936-7628

E-mail: vlpec@umich.edu

Prof. J. A. Stuckey

Life Sciences Institute, University of Michigan

Ann Arbor, MI 48109 (USA)

[***] V.L.P. thanks the National Institutes of Health for support of this research (ES012236), and J.A.S. thanks the University of Michigan Center for Structural Biology.

믄 Supporting information for this article is available on the WWW under http://dx.doi.org/10.1002/anie.200902166. these peptides are diastereopeptides, this stereochemical modification is occurring within a sea of L-amino acids. These issues could be best addressed by performing comparative spectroscopic and structural studies on three-stranded coiled coils containing either L-Pen or D-Pen (see Figure S1 in the Supporting Information). We chose to perform experiments on the related peptide Coil Ser (CS) ${ }^{[11]}$ Ac-E WEALEKK LAALESK LQALEKK LEALEHG-NH ${ }_{2}$, with Land D-Pen substituted at the 16-position (CSL16L-Pen and CSL16D-Pen), as Coil Ser behaves similarly to the TRI family, with which the majority of studies have been carried out, ${ }^{[12]}$ yet has proven to be more readily crystallizable..$^{[13,14]}$

First, we explored the interactions of these peptides in aqueous solution with $\mathrm{Cd}^{\mathrm{II}}$. ${ }^{113} \mathrm{Cd} \mathrm{NMR}$ spectroscopy is a powerful tool for determining the coordination environment of $\mathrm{Cd}^{\mathrm{II}} \cdot{ }^{[15]}$ The ${ }^{113} \mathrm{Cd} \mathrm{NMR}$ spectrum for $\mathrm{Cd}^{\mathrm{II}}$ bound to the L-amino acid analogue of Pen, CSL16L-Pen, shows a single resonance at $\delta=683 \mathrm{ppm}$. This signal is almost identical to that reported previously for the TRI analogue $(\delta=684 \mathrm{ppm})$ and thus confirms that $\mathrm{Cd}^{\mathrm{II}}$ is bound as a trigonal-planar three-coordinate species. ${ }^{[8]}$ For the D-Pen analogue, a resonance of weaker intensity was observed at $\delta=557 \mathrm{ppm}$, which is well outside the range associated with $\mathrm{CdS}_{3}(\delta=680-$ $700 \mathrm{ppm}$ ) and more consistent with an $\mathrm{S}_{2} \mathrm{O}_{2}$-type (or possibly $\mathrm{S}_{3} \mathrm{O}$-type) first-coordination-sphere environment (see Figure S2 in the Supporting Information). ${ }^{[15]}$

The binding of $\mathrm{Cd}^{\mathrm{II}}$ to these constructs was also monitored by $\mathrm{UV} / \mathrm{Vis}$ spectroscopy and was once again found to be different for the L- and D-Pen analogues (see Figure S3 in the Supporting Information). The characteristic ligand-to-metal charge transfer (LMCT) band for $\mathrm{Cd}^{\mathrm{II}}$ bound to L-Pen as $\mathrm{Cd}(\mathrm{CSL} 16 \mathrm{~L}-\mathrm{Pen})_{3}{ }^{-}$is similar $\quad\left(\lambda_{\max }=227 \mathrm{~nm}, \quad \varepsilon_{227}=\right.$ $36000 \mathrm{M}^{-1} \mathrm{~cm}^{-1}$ ) to that reported for Cd(TRIL16L-Pen) ${ }_{3}{ }^{-} \cdot{ }^{[16]}$ In contrast, an absorption band at $\lambda_{\max } \approx 210 \mathrm{~nm}$ was observed for solutions containing $\mathrm{Cd}^{\mathrm{II}}$ in the presence of CSL16D-Pen at $\mathrm{pH} 9.6$, a $\mathrm{pH}$ value at which we generally expect $\mathrm{Cd}^{\mathrm{II}}$ to be fully bound.

To determine the $\mathrm{pH}$ dependence associated with $\mathrm{Cd}^{\mathrm{II}}$ binding, we monitored absorbance as a function of $\mathrm{pH}$ value. The resulting $\mathrm{pH}$ titration curves (see Figure $\mathrm{S} 4$ in the Supporting Information) reveal that $\mathrm{Cd}^{\mathrm{II}}$ binds to CSL16LPen with a high $\mathrm{p} \mathrm{Ka}_{2}$ value (16.3), which is consistent with the behavior of $\mathrm{Cd}^{\mathrm{II}}$ binding to a three-coordinate site. ${ }^{[15,16]}$ However, no significant increase in absorbance was observed for CSL16 D-Pen until the $\mathrm{pH}$ value was greater than 9, at which point unfolding of the apo peptide contributes to the signal (see Figure S4 in the Supporting Information). This behavior is not consistent with $\mathrm{Cd}^{\mathrm{II}}$ bound to three thiolate groups (either $\mathrm{CdS}_{3}$ or $\mathrm{CdS}_{3} \mathrm{O}$ ). However, it would be 
consistent with a more sulfur poor coordination environment, such as $\mathrm{CdS}_{2} \mathrm{O}_{2}$ (or $\mathrm{CdSO}_{3}$ ).

Although the first coordination sphere of the $\mathrm{Cd}^{\mathrm{II}}$ ion is not chiral when the ion is bound in the interior of these coiled coils, the peptide environment conveys chirality onto the metal, which results in distinct CD (circular dichroism) difference spectra. Similar transitions, both with negative intensity at $257 \mathrm{~nm}$, are present in the CD difference spectra when $\mathrm{Cd}^{\mathrm{II}}$ is bound to CSL16L-Pen or TRIL16L-Pen. ${ }^{[17]}$ No transition was observed in analogous experiments performed with CSL16D-Pen (see Figure S5 in the Supporting Information).

The combination of ${ }^{113} \mathrm{Cd} \mathrm{NMR}$, UV/Vis, and CD spectroscopy supports the conclusion that $\mathrm{Cd}^{\mathrm{II}}$ binds as a trigonal three-coordinate species to L-Pen-substituted Coil Ser. In contrast, $\mathrm{Cd}^{\mathrm{II}}$ binds to $\mathrm{D}-\mathrm{Pen}$ in a radically different manner: only a small fraction of $\mathrm{Cd}^{\mathrm{II}}$ binds to D-Pen-substituted Coil Ser, and it binds with a mixed first coordination sphere, which is probably four-coordinate $\mathrm{CdS}_{2} \mathrm{O}_{2}$ (each $\mathrm{O}$ is an exogenous water molecule).

Clearly, metal binding is very different upon inversion of the chirality of a layer of amino acid ligands in the interior of a three-stranded coiled coil. Before these data are interpreted fully, it is important to understand how an inversion of chirality changes the peptide construct in the absence of metal ions. CD spectra demonstrate that the construct with a layer of L-Pen residues forms a well-folded coiled coil (negative maxima at 208 and $222 \mathrm{~nm} ;>90 \%$ folded). Although the ellipticity at $222 \mathrm{~nm}$ is still indicative of a right-handed $\alpha$-helix when this ligand is replaced with D-Pen, it decreases in intensity, which suggests that CSL16D-Pen is less well folded (ca. 50\%) than the L-Pen analogue (see Figure S6A in the Supporting Information). Consistent with this conclusion, CSL16D-Pen is less stable toward denaturant (guanidine hydrochloride) than CSL16L-Pen; the relative half points are at denaturant concentrations of approximately 0.4 and $1.5 \mathrm{M}$, respectively (see Figure S6 B in the Supporting Information). It has been reported that the introduction of a single D-Ala residue onto the solvent-exposed face of Coil Ser to form CSL14D-Ala destabilizes the helix in a two-stranded coiled coil by $0.95 \mathrm{kcal} \mathrm{mol}^{-1} \cdot{ }^{[18,19]}$ Shorter HPLC retention times (by 0-10 min) for D-amino acid containing peptides relative to the retention times of the equivalent peptides composed only of L-amino acids have been reported to correlate with the destabilizing effect of the substitution (HPLC retention times are related to the $\alpha$-helical content of the peptide). ${ }^{[20-22]}$ Consistent with this trend, we found that CSL16D-Pen (30 min) was eluted faster than CSL16L-Pen (33 min). The helix-destabilizing propensity of D-amino acids has been shown to be highly dependent on the size of the side chain, whereby $\beta$-branched amino acids (a category to which D-Pen would belong) are the most effective. ${ }^{[20,21]}$ The substitution of $\mathrm{D}$-Val in the center of a 19 amino acid $\alpha$-helix results in a significantly less $\alpha$-helical structure $(50 \%)$ relative to that of the $\mathrm{L}$-Val analogue $(>90 \%),{ }^{[23]}$ whereas D-Leu residues (without $\beta$ branches) are only medium helix destabilizers, in agreement with our previous observations. ${ }^{[9]}$

$\mathrm{X}$-ray crystal structures of $\mathrm{D}$-amino acid residues in sequences containing predominantly L-amino acids are rare, especially in longer polypeptides of 20 residues or more. ${ }^{[24]}$ Furthermore, such structures of peptides containing an $\mathrm{L}-\mathrm{D}-\mathrm{L}$ sequence offer the potential to evaluate conformations that would be accessible to a D-amino acid residue within a natural protein environment. Although heteromeric coiled coils consisting of bundles containing either all L- or all D-helices have been reported, ${ }^{[25]}$ to the best of our knowledge, no reports exist on structures of L-amino acid coiled coils containing a single layer of D-amino acids. Despite the differences in stability, both CSL16L-Pen and CSL16D-Pen crystallized in the $C_{2}$ space group. Their structures were solved to 1.86 and $1.71 \AA$ resolution, respectively. Both structures are well-folded three-stranded coiled coils with the $\alpha$-helices oriented parallel to one another (Figure 1).
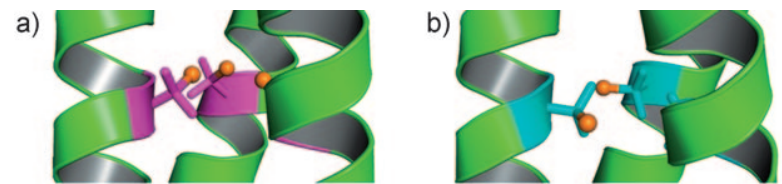

c)
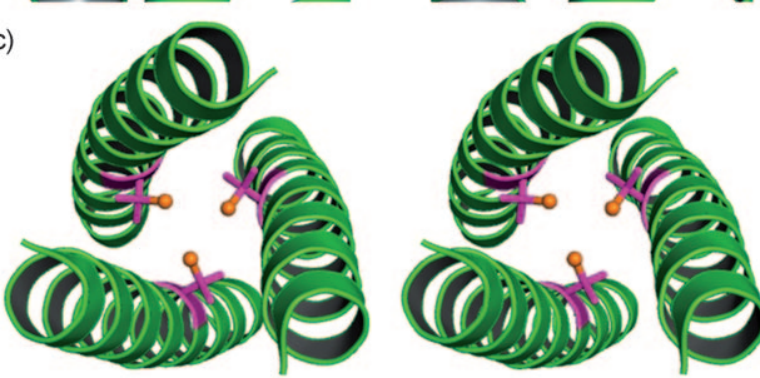

d)
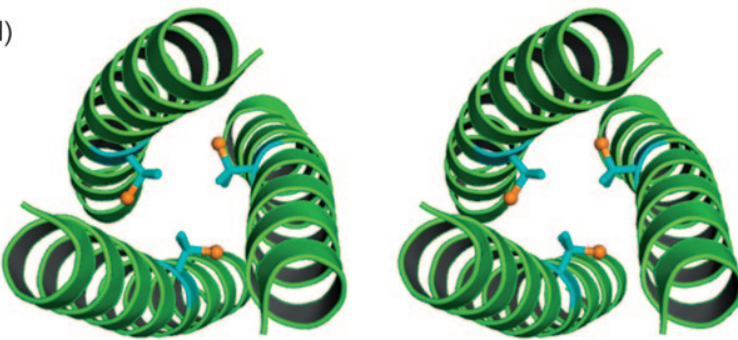

Figure 1. Ribbon diagrams of the CSL16X-Pen trimer showing the orientation of the X-Pen side chain. Shown are the main chain atoms represented as helical ribbons (green) and the L- and D-Pen side chain in stick form (L-Pen pink; D-Pen blue) with the thiol group shown as a sphere (orange) in the interior of the parallel three-stranded coiled coil. Side views show the a) L-Pen and b) D-Pen side chains oriented towards the $\mathrm{N}$ and $\mathrm{C}$ terminus, respectively. A stereo top-down view from the $\mathrm{N}$ terminus of the coiled coil is shown for $\mathrm{c}$ ) L-Pen (major rotamer, $95 \%$ ) and d) D-Pen.

L-Pen ( $\beta, \beta$-dimethylcysteine), often used in de novo peptide design, ${ }^{[7,8,16]}$ is considered to be a bulkier analogue of L-Cys. To the best of our knowledge, L-Pen has not been characterized crystallographically in a protein environment. Following our success in creating trigonal $\mathrm{CdS}_{3}$ through bonding to L-Pen, ${ }^{[8,16]}$ we realized it was crucial to determine precisely what this nonprotein amino acid looks like within the interior of a coiled coil. To build the L-Pen residue, we started from a model structure containing L-Cys. After one round of refinement, electron density for the methyl groups of L-Pen was clearly present in the $F_{\mathrm{o}}-F_{\mathrm{c}}$ map (see Figure S7 A 
in the Supporting Information). The L-Pen ligand could then be built by using this density, by placing the methyl groups at the positions of the L-Cys $\beta$-methylene hydrogen atoms. Alternatively, one can envision L-Pen as a thiol-containing analogue of $\mathrm{L}$-valine $(\mathrm{L}-\mathrm{Val})$ in which the single $\beta$-methylene hydrogen atom is replaced by a thiol group ( $\beta$-mercaptovaline). When we used a rotamer of $\mathrm{L}$-Val in the model, we observed electron density consistent with a thiol group in the $F_{\mathrm{o}}-F_{\mathrm{c}}$ map (see Figure S7B in the Supporting Information). The resulting side chain of L-Pen is oriented towards the $\mathrm{N}$ terminus in the interior of the coiled coil (Figure 1 A) in a similar manner to L-Cys. The thiol group of the major rotamer $(95 \%)$ is positioned towards the interior of the coiled coil (S-S distances of $3.7 \AA$ ) as if preorganized for metal binding (Figure $1 \mathrm{C}$ ). Intriguingly, the methyl groups can be divided into two distinct classes: those situated above the plane (towards the $\mathrm{N}$ terminus) appear to be oriented away from the helical axis and towards the helical interfaces, whereas those positioned below the thiol-containing plane point towards the interior of the coiled coil and block this face. This structure suggests that the role of the methyl groups may be to enable the existence of a coordinatively unsaturated $\mathrm{CdS}_{3}$ species by improving packing and preventing access to the site. In the lower-abundance conformation (5\%), one of the thiol side arms is rotated so that the two methyl groups point towards the interior of the coiled coil and the sulfur atom towards the helical interface (see Figure S8 in the Supporting Information).

The structure of the D-Pen side chain was determined as described for L-Pen, by using a model based on D-Cys (or $\mathrm{D}$-Val). The introduction of a single D-amino acid into an all L-amino acid coiled coil does not lead to a major perturbation of the $\alpha$-helical backbone; thus, the two structures overlay very well (see Figure S9 in the Supporting Information). This observation is important, as D-amino acids are commonly used to induce turns or terminate designed $\alpha$-helices. ${ }^{[26-29]}$ All non-glycine residues (including D-Pen) fall within the preferred region of the Ramachandran plot for $\alpha$-helices (see Figure S10 in the Supporting Information). ${ }^{[30]}$ This observation is consistent with reported X-ray crystal structures of predominantly L-amino acid $\alpha$-helices, which have been shown to tolerate up to three D-amino acids in a 19 amino acid sequence. ${ }^{[31,32]}$ Any energy penalty associated with misorientation of the side chain in a left-handed $\alpha$-helix must be offset by favorable total hydrophobic-packing interactions and the intramolecular hydrogen bonds formed along the $\alpha$-helical backbone. The (CSL16D-Pen) ${ }_{3}$ structure shows that switching of the chirality of an internal amino acid and reorientation of the $\mathrm{D}$-Pen side chain towards the $\mathrm{C}$ terminus (Figure 1B) does not prevent the three-stranded coiled coil from assembling. The addition of at least one (sterically bulky) D-amino acid is well-tolerated in such constructs and is likely to be tolerated in analogous designed coiled coils.

The thiol group in D-Pen is oriented towards the C terminus, away from the interior, and directed towards the helical interface (S-S distances of $6.5 \AA$ ), whereas both methyl groups are now positioned within the hydrophobic interior of the coiled coil (Figure 1D). Although the major rotamer of L-Pen appears to be more closely related to $\beta, \beta$-dimethylcysteine, the D-Pen residue behaves more like the thiol-containing analogue of $\mathrm{D}$-Val. In contrast to that of L-Pen (Figure 1C), this site is not preorganized for metal binding and is incapable of forming $\mathrm{CdS}_{3}$ or $\mathrm{CdS}_{3} \mathrm{O}$ forms within the coiled coil. However, there is evidence that a small amount of $\mathrm{Cd}^{\mathrm{II}}$ is capable of binding, most likely as $\mathrm{CdS}_{2} \mathrm{O}_{2}$. This structure may be formed through the orientation of the thiol groups of two D-Pen ligands towards the same helical interface; $\mathrm{Cd}^{\mathrm{II}}$ could then bind to the two thiol ligands as well as two molecules of solvent (water). The Pen side chain resembles a propeller with bulky blades (three groups on the $\beta$ carbon atom), which may not rotate readily. In the case of D-Pen, which does not provide a preorganized metal-binding site, the energy penalty for repositioning the thiol groups so as to be favorable for metal binding may be too high. Scheme 1 illustrates the two probable metal-binding modes in $\mathrm{L}-$ and $\mathrm{D}-\mathrm{Pen}$.

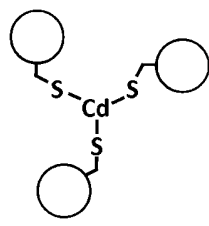

a) L-Pen

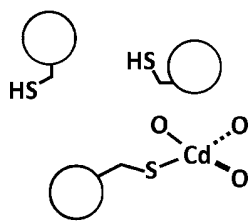

b) D-Pen

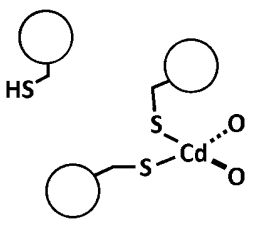

c) D-Pen
Scheme 1. A) ChemDraw representation of the established $\mathrm{CdS}_{3}$ binding to L-Pen in the interior of a three-stranded coiled coil. Two possible

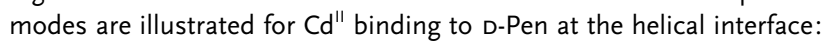
B) $\mathrm{CdSO}_{3}$ or C) $\mathrm{CdS}_{2} \mathrm{O}_{2}$. The latter $\left(\mathrm{CdS}_{2} \mathrm{O}_{2}\right)$ would require reorientation of one D-Pen residue for $\mathrm{Cd}^{\prime \prime}$ binding.

The results of this study provide new strategies for protein design by highlighting the relationship between L- and D-Pen as analogues of Cys and Val. Previously, we showed that one can control $\mathrm{Cd}^{\mathrm{II}}$ coordination geometry by modifying the chirality of a second-coordination-sphere ligand. ${ }^{[9]}$ Herein, we showed that a change in the chirality of the coordinating ligand can not only drastically alter the coordination environment of the metal within a protein, but may change the physical location of the metal ion within the construct.

$\mathrm{X}$-ray crystallography suggests that metal-binding differences may arise because D-Pen may behave like a pseudo D-Val residue, whereas L-Pen behaves more like L-Cys (with a preorganized metal-binding site). We have shown that the incorporation of $\mathrm{D}$-amino acids into predominantly L-amino acid coiled coils is well-tolerated; thus, the broad application of D-amino acids in the field of protein design should be possible.

\section{Experimental Section}

Peptide synthesis: Peptides were synthesized on an Applied Biosystem $433 \mathrm{~A}$ peptide synthesizer by using standard protocols ${ }^{[33]}$ and characterized by electrospray mass spectrometry. Peptides were purified by preparative reversed-phase C18 HPLC at a flow rate of $10 \mathrm{~mL} \mathrm{~min}^{-1}$ with a solvent mixture altered with a linear gradient from $0.1 \%$ trifluoroacetic acid (TFA) in water to $0.1 \%$ TFA in $\mathrm{CH}_{3} \mathrm{CN} / \mathrm{H}_{2} \mathrm{O}(9: 1)$ over $50 \mathrm{~min}$. Stock solutions were prepared in 
degassed doubly distilled water, and their concentrations were determined by using a previously described assay. ${ }^{[34]}$

${ }^{113} \mathrm{Cd}$ NMR spectra were collected and data processed as reported previously. ${ }^{[9]}$

$\mathrm{CD}$ and UV/Vis spectroscopy: $\mathrm{CD}$ and UV/Vis spectra were recorded in quartz cuvettes at $298 \mathrm{~K}$ on an AVIV 62DS spectrometer and Cary 100 Bio UV/Vis spectrometer, respectively. The observed CD ellipticity in millidegrees was converted into molar ellipticity, $[\Theta]$, and is reported in units of $\mathrm{deg} \mathrm{dmol}^{-1} \mathrm{~cm}^{2}$. Difference spectra were obtained by subtracting the background spectrum of the peptide in the absence of a metal. $\mathrm{pH}$ titrations were performed as reported previously, and the experimental data for CSL16D-Pen was fit to a model for the simultaneous release of two protons on the binding of $\mathrm{Cd}^{\mathrm{II}}$ to three thiolate groups. ${ }^{[9,35]}$ Guanidine hydrochloride CD titrations were performed at $\mathrm{pH} 8.5$ as described previously. ${ }^{[3]}$

Crystallization: Crystals of CSL16L-Pen were grown by vapor diffusion at $20^{\circ} \mathrm{C}$ in a sitting drop with equal volumes of peptide (20 $\mathrm{mg} \mathrm{mL}^{-1}$ CSL16L-Pen, $2.0 \mathrm{~mm} \mathrm{CdCl}_{2}, 20 \mathrm{~mm} \mathrm{Zn}(\mathrm{OAc})_{2}, 5 \mathrm{~mm}$ Tris buffer, $\mathrm{pH} 8.5)$ and precipitant (100 mM cacodylate, $\mathrm{pH} 6.5$, $200 \mathrm{~mm} \mathrm{MgCl}_{2}, 50 \%$ PEG 200)). Crystals of CSL16D-Pen were grown by vapor diffusion at $20^{\circ} \mathrm{C}$ in a hanging drop with equal volumes of peptide $\left(15.8 \mathrm{mg} \mathrm{mL}^{-1}\right.$ CSL16D-Pen, $8.4 \mathrm{~mm} \mathrm{CdCl}_{2}, 8.4 \mathrm{~mm} \mathrm{Zn-}$ $(\mathrm{OAc})_{2}, 5 \mathrm{~mm}$ Tris buffer, $\left.\mathrm{pH} 8.5\right)$ and precipitant (100 mM MES pH 6.0, 30\% PEG 200, 5 \% PEG 3000). Crystals were frozen in their mother liquor for data collection.

Crystal-data collection: Data were collected at the Advanced Photon Source of the Argonne National Laboratory on the LS-CAT Beamline 21-ID equipped with a Mar 225 CCD detector (Mar USA, Evanston, IL). A total of 360 frames of data were collected at $-180^{\circ} \mathrm{C}$ with a $1^{\circ}$ rotation and $2 \mathrm{~s}$ exposure. Data were processed and scaled with the program HKL-2000. ${ }^{[36]}$ The structures were solved by molecular replacement with Phaser in the CCP4 suite of programs $^{[37,38]}$ by using as a model As(CSL9C) ${ }_{3}$ (Protein Data Bank ID 2 jgo $)^{[14]}$ with the metal ions removed but the side chains included. These models were refined with restrained refinement in Refmac 5 in the CCP4 suite of programs ${ }^{[39]}$ and built in $\mathrm{Coot}^{[40]}$ by using the $2 F_{\mathrm{o}}-F_{\mathrm{c}}$ and $F_{\mathrm{o}}-F_{\mathrm{c}}$ electron-density maps generated in Refmac 5 . The structures of CSL16L-Pen and CSL16D-Pen were refined to $1.86 \AA$ $\left(R_{\text {working }}=19.9 \%, R_{\text {free }}=25.6 \%\right)$ and $1.71 \AA\left(R_{\text {working }}=18.1 \%, R_{\text {free }}=\right.$ $22.5 \%)$, respectively. Data collection and refinement statistics are given in Table S1 in the Supporting Information. The atomic coordinates and structure factors for CSL16L-Pen and CSL16D-Pen have been deposited in the Protein Data Bank with the ID codes $3 \mathrm{H} 5 \mathrm{~F}$ and $3 \mathrm{H} 5 \mathrm{G}$, respectively. Figures were generated in PyMOL. ${ }^{[41]}$

Received: April 22, 2009

Published online: July 3, 2009

Keywords: cadmium · crystallography · D-amino acids · de novo peptides $\cdot$ protein design

[1] W. F. DeGrado, C. M. Summa, V. Pavone, F. Nastri, A. Lombardi, Annu. Rev. Biochem. 1999, 68, 779.

[2] D. Ghosh, V. L. Pecoraro, Inorg. Chem. 2004, 43, 7902.

[3] G. R. Dieckmann, D. K. McRorie, J. D. Lear, K. A. Sharp, W. F. DeGrado, V. L. Pecoraro, J. Mol. Biol. 1998, $280,897$.

[4] M. K. Yadav, J. E. Redman, L. J. Leman, J. M. Alvarez-Gutiérrez, Y. Zhang, C. D. Stout, M. R. Ghadiri, Biochemistry 2005, 44, 9723.

[5] K.-H. Lee, M. Matzapetakis, S. Mitra, E. N. G. Marsh, V. L. Pecoraro, J. Am. Chem. Soc. 2004, 126, 9178.

[6] G. R. Dieckmann, D. K. McRorie, D. L. Tierney, L. M. Utschig, C. P. Singer, T. V. O'Halloran, J. E. Penner-Hahn, W. F. DeGrado, V. L. Pecoraro, J. Am. Chem. Soc. 1997, 119, 6195.

[7] A. K. Petros, S. E. Shaner, A. L. Costello, D. L. Tierney, B. R Gibney, Inorg. Chem. 2004, 43, 4793.
[8] K.-H. Lee, C. Cabello, L. Hemmingsen, E. N. G. Marsh, V. L. Pecoraro, Angew. Chem. 2006, 118, 2930; Angew. Chem. Int. Ed. 2006, 45, 2864.

[9] A. F. A. Peacock, L. Hemmingsen, V. L. Pecoraro, Proc. Natl. Acad. Sci. USA 2008, 105, 16566

[10] A. F. A. Peacock, O. Iranzo, V. L. Pecoraro, J. Chem. Soc. Dalton Trans. 2009, 2271.

[11] K. T. O’Neil, W. F. DeGrado, Science 1990, 250, 646.

[12] O. Iranzo, D. Ghosh, V. L. Pecoraro, Inorg. Chem. 2006, 45, 9959.

[13] B. Lovejoy, S. Choe, D. Cascio, D. K. McRorie, W. F. DeGrado, D. Eisenberg, Science 1993, 259, 1288.

[14] D. S. Touw, C. E. Nordman, J. E. Stuckey, V. L. Pecoraro, Proc. Natl. Acad. Sci. USA 2007, 104, 11969.

[15] O. Iranzo, M. Stachura, T. Jakusch, K.-H. Lee, L. Hemmingsen, V. L. Pecoraro, Chem. Eur. J. 2007, 13, 9178.

[16] O. Iranzo, C. Cabello, V. L. Pecoraro, Angew. Chem. 2007, 119, 6808; Angew. Chem. Int. Ed. 2007, 46, 6688.

[17] O. Iranzo, V. L. Pecoraro, unpublished results.

[18] R. Fairman, S. J. Anthony-Cahill, W. F. DeGrado, J. Am. Chem. Soc. 1992, 114, 5458.

[19] J. Hermans, A. G. Anderson, R. H. Yun, Biochemistry 1992, 31, 5646.

[20] E. Krause, M. Bienert, P. Schmieder, H. Wenschuh, J. Am. Chem. Soc. 2000, 122, 4865.

[21] Y. Chen, C. T. Mant, R. S. Hodges, J. Pept. Res. 2002, 59, 18.

[22] H. Wendt, C. Berger, A. Baici, R. M. Thomas, H. R. Bosshard, Biochemistry 1995, 34, 4097.

[23] E. Krause, M. Beyermann, M. Dathe, S. Rothemund, M. Bienert, Anal. Chem. 1995, 67, 252.

[24] J. B. O. Mitchell, J. Smith, Proteins Struct. Funct. Genet. 2003, 50, 563.

[25] S. K. Sia, P. S. Kim, Biochemistry 2001, 40, 8981.

[26] T. Haack, M. J. González, Y. Sánchez, E. Giralt, Lett. Pept. Sci. 1997, 4, 377.

[27] S. Aravinda, N. Shamala, R. S. Roy, P. Balaram, Proc. Indian Acad. Sci. Chem. Sci. 2003, 115, 373.

[28] "The use of D-amino acids in peptide design": R. Mahalakshmi, P. Balaram in D-Amino Acids: A New Frontier in Amino Acid and Protein Research (Eds.: R. Konno, G. H. Fisher, H. Brueckner, A. d'Aniello, N. Fujii, H. Homma), Nova Science, NY, 2006, pp. $415-430$.

[29] S. Durani, Acc. Chem. Res. 2008, 41, 1301

[30] G. N. Ramachandran, V. Sasisekharan, Adv. Protein. Chem. 1968, 23, 283.

[31] S. Aravinda, N. Shamala, S. Desiraju, P. Balaram, J. Chem. Soc. Chem. Commun. 2002, 2454.

[32] I. L. Karle, H. N. Gopi, P. Balaram, Proc. Natl. Acad. Sci. USA 2003, 100, 13946.

[33] Fmoc Solid Phase Peptide Synthesis: A Practical Approach (Eds.: W. C. Chan, P. D. White), Oxford University Press, New York, 2000, p. 346.

[34] M. Mantle, G. Stewart, G. Zayas, M. King, Biochem. J. 1990, 266, 597.

[35] M. Matzapetakis, D. Ghosh, T.-C. Weng, J. E. Penner-Hahn, V. L. Pecoraro, J. Biol. Inorg. Chem. 2006, 11, 876.

[36] Z. Otwinowski, W. Minor, Enzymology 1997, 276, 307.

[37] E. Potterton, P. Briggs, M. Turkenburg, E. Dodson, Acta Crystallogr. Sect. D 2003, 59, 1131.

[38] A. J. McCoy, R. W. Grosse-Kunstleve, P. D. Adams, M. D. Winn, L. C. Storoni, R. J. Read, J. Appl. Crystallogr. 2007, 40, 658.

[39] G. N. Murshudov, A. A. Vagin, E. J. Dodson, Acta Crystallogr. Sect. D 1997, 53, 240.

[40] P. Emsley, K. Cowtan, Acta Crystallogr. Sect. D 2004, 20, 2126.

[41] W. L. Delano, The PyMOL Molecular Graphics System, DeLano Scientific, Palo Alto, California, USA, 2005. http:// www.pymol.org. 